\title{
Estudio Numérico de la Resistencia Térmica en Muros de Bloques de Concreto Hueco con Aislamiento Térmico
}

\author{
Jesús B. Pérez, Rafael E. Cabanillas, Jesús F. Hinojosa, Ana C. Borbón \\ División de Ingeniería, Universidad de Sonora, Rosales y Boulevard Luis Encinas, Colonia Centro, \\ CP 83000, Hermosillo Sonora, México (e-mail: benito@dicym.uson.mx, rcabani@iq.uson.mx, \\ fhinojosa@iq.uson.mx, acborbon@dicym.uson.mx)
}

Recibido Ago. 04, 2010; Aceptado Oct. 13, 2010; Versión Final recibida Nov. 11, 2010

\begin{abstract}
Resumen
Se presenta el estudio numérico de la resistencia térmica en dos tipos de muros de bloques de concreto hueco para diferentes temperaturas en la superficie exterior. Se analiza el efecto de colocar aislamiento térmico por la cara exterior y en sus cavidades. El modelo teórico para estado permanente, fue resuelto numéricamente con diferencias finitas y validado con resultados experimentales de la literatura. Las resistencias térmicas promedio para los bloques de $0.12 \mathrm{~m}$ y $0.15 \mathrm{~m}$ son de $0.159 \mathrm{~m}^{2} \cdot \mathrm{K} / \mathrm{W}$ y $0.171 \mathrm{~m}^{2} \cdot \mathrm{K} / \mathrm{W}$ respectivamente. Cuando se rellenan las cavidades con aislamiento térmico son de $0.407 \mathrm{~m}^{2} \mathrm{~K} / \mathrm{W}$ y de $0.530 \mathrm{~m}^{2} \cdot \mathrm{K} / \mathrm{W}$, respectivamente. Al aplicar el mismo volumen de aislante de la cavidad rellena, sobre la cara exterior, se obtienen valores de $1.555 \mathrm{~m}^{2} \cdot \mathrm{K} / \mathrm{W}$ y $2.147 \mathrm{~m}^{2} \cdot \mathrm{K} / \mathrm{W}$, para los bloques de $0.12 \mathrm{~m}$ y $0.15 \mathrm{~m}$ respectivamente.
\end{abstract}

Palabras clave: resistencia térmica, bloque concreto hueco, aislamiento térmico, diferencias finitas

\section{Numerical Study of the Thermal Resistance of Hollow Concrete Block Walls with Thermal Insulation}

\begin{abstract}
A numerical study of the thermal resistance in walls made of two different hollow concrete blocks is presented varying the external surface temperature. The effect of thermal insulation position, on the external concrete block surface and in the cavities, was analyzed. The theoretical model for the steady state was solved numerically with the finite differences technique and was compared with experimental results reported in literature. The thermal resistances for $0.12 \mathrm{~m}$ and $0.15 \mathrm{~m}$ blocks are $0.159 \mathrm{~m}^{2} \cdot \mathrm{K} / \mathrm{W}$ and $0.171 \mathrm{~m}^{2} \cdot \mathrm{K} / \mathrm{W}$, respectively. The maximum thermal resistances after filling the cavities with thermal insulation are $0.407 \mathrm{~m}^{2} \cdot \mathrm{K} / \mathrm{W}$ and $0.530 \mathrm{~m}^{2} \cdot \mathrm{K} / \mathrm{W}$ for $0.12 \mathrm{~m}$ and $0.15 \mathrm{~m}$ blocks respectively. When the same volume of insulation is applied on the external surface, the thermal resistance values are $1.555 \mathrm{~m}^{2} \mathrm{~K} / \mathrm{W}$ and $2.147 \mathrm{~m}^{2} \cdot \mathrm{K} / \mathrm{W}$ for $0.12 \mathrm{~m}$ and $0.15 \mathrm{~m}$ blocks respectively.
\end{abstract}

Keywords: thermal resistance, concrete hollow block, thermal insulation, finite difference 


\section{INTRODUCCIÓN}

El principal problema de la construcción de viviendas con bloques de concreto hueco, es su baja resistencia térmica, ya que esto ocasiona que en climas extremosos las ganancias de calor sean elevadas y como consecuencia las condiciones de habitabilidad intramuros se encuentren fuera de la zona de confort.

El boque de concreto utilizado en México es fabricado en diversas medidas, los más comunes que se utilizan en la construcción de viviendas son de $15 \times 20 \times 40$ y $12 \times 20 \times 40$ centímetros, con dos cavidades huecas interiores de 10×16 y $7 \times 16$ centímetros respectivamente (fig. 1). La forma en que se disponen los bloques huecos al fabricar los muros, forman cavidades alargadas verticales en forma de ductos con altura mínima de $2 \mathrm{~m}$, denominada altura de cerramiento sin considerar la junta de mortero, asimismo tienen una sección transversal máxima de $10 \times 16 \mathrm{~cm}$ para el bloque de $15 \mathrm{~cm}$; de $7 \times 16 \mathrm{~cm}$ para el bloque de $12 \mathrm{~cm}$ de ancho (fig. 2). La determinación de la resistencia térmica en muros con base en bloques huecos, involucra la consideración de los mecanismos acoplados de transferencia de calor por conducción a través del concreto, así como la convección natural y radiación en las cavidades.

En la literatura especializada se han publicado varios estudios de la resistencia térmica en muros de mampostería, descritos a continuación.

Anderson (1981) realizó un estudio numérico de la resistencia térmica en bloques huecos de concreto, aplicando el método de elemento finito, encontrando valores de la resistencia térmica de alrededor de $0.250 \mathrm{~m}^{2} \mathrm{~K} / \mathrm{W}$, para las dimensiones y configuraciones consideradas. Por su parte, Ossama y Kris (1994) evalúan experimentalmente la resistencia térmica en un muro de bloque de concreto hueco de $0.150 \mathrm{~m}$ de ancho con base en la prueba ASTM C 518-76, el muro evaluado térmicamente es equivalente al bloque de $0.150 \mathrm{~m}$ de nuestro estudio; se reportan valores de resistencia térmica de $0.331 \mathrm{~m}^{2} \mathrm{~K} / \mathrm{W}$ sin considerar la influencia del mortero y de $0.361 \mathrm{~m}^{2} \mathrm{~K} / \mathrm{W}$ cuando los bloques se juntan con mortero.

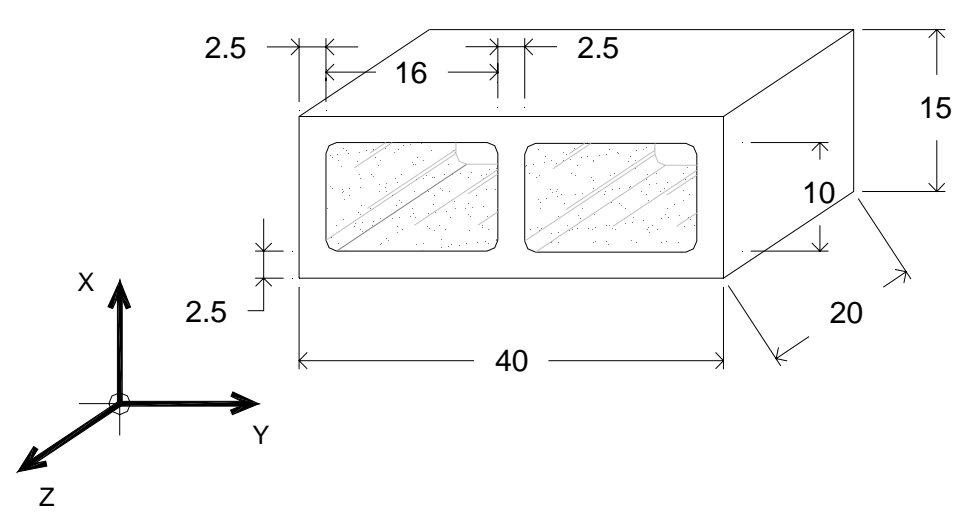

Fig 1, Medidas nominales de un bloque de concreto en centímetros.

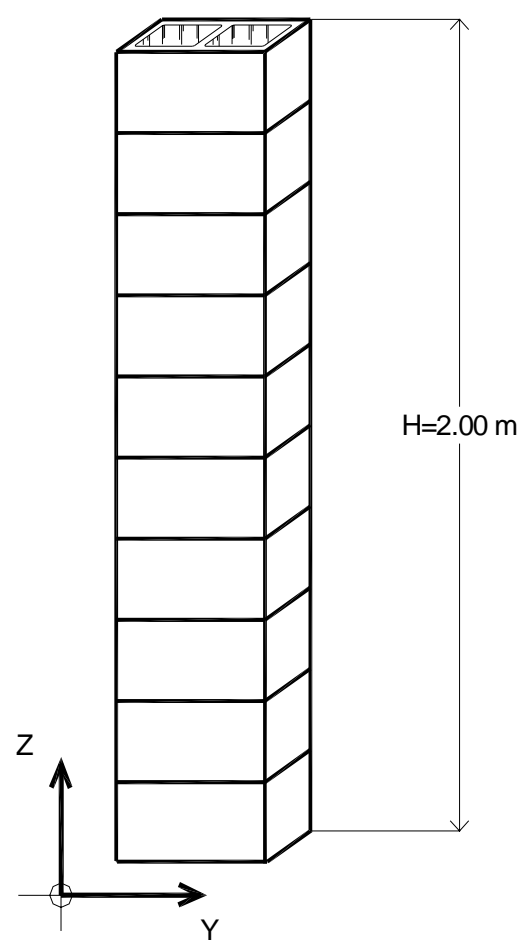

Fig 2, Cavidad alargada constructiva. 
Lorente y colaboradores (1996) encontraron mediante un estudio teórico y experimental, que el $25 \%$ del total de calor transferido fue por convección, otro $25 \%$ por conducción y $50 \%$ por radiación. Vasile y colaboradores (1998) realizaron un estudio teórico en ladrillos de terracota huecos donde se encuentra que la resistencia térmica disminuye con respecto al aumento en la diferencia de temperatura. Aviram y colaboradores (2001) en estudios experimentales demostraron que los flujos de calor disminuyen al aumentar la razón de aspecto de la cavidad hueca. Wakili y Tanner (2003) caracterizan un muro de $0.40 \times 1.56 \times 1.49 \mathrm{~m}$ con tabiques de arcilla multiperforados de forma experimental y numérica; reportando valores sobreestimados en la modelación del orden 3 al $5 \%$ con respecto a la mediciones experimentales. Lacarrie 're y colaboradores (2003) encontraron a través de modelación numérica que las perforaciones del ladrillo de arcilla favorecen la resistencia térmica y cuando se usa mortero para la unión de los tabiques, se crean zonas de menor resistencia térmica.

Majed M. Al-Hazmy (2006) reporta el estudio de la transferencia de calor por convección natural y conducción acoplados, en un bloque con tres cavidades huecas, y encuentra que los huecos llenos de aire del bloque, contribuyen a la transferencia de calor, encontrándose que esta se reduce en un máximo de $36 \%$ si se rellenan con poliestireno común. Lacarrie 're y colaboradores (2006) estudiaron el desempeño térmico en estado transitorio de la transferencia de calor en un pieza de ladrillo de arcilla multiperforado de forma experimental. Borbón y colaboradores (2010) realizan un estudio experimental de la resistencia térmica de un muro de bloque de concreto hueco de $12 \times 20 \times 40$ centímetros, $2.00 \mathrm{~m}$ de alto, por $1.60 \mathrm{~m}$ de ancho, reproduciendo las condiciones ambientales para la ciudad Hermosillo, Sonora, en épocas de sobrecalentamiento; para mediciones en estado permanente se reporta una resistencia térmica promedio de 0.174 $\mathrm{m}^{2} \mathrm{~K} / \mathrm{W}$.

En el presente trabajo se obtienen valores de la resistencia térmica en un muro de bloque de concreto hueco, utilizando la técnica numérica de diferencias finitas para resolver el modelo matemático planteado. Los resultados son validados con datos experimentales y se evalúa el comportamiento de la resistencia térmica, al utilizar diferentes alternativas de colocación de aislamiento térmico en un muro de bloque con concreto hueco.

\section{MODELO MATEMÁTICO}

El problema de transporte de energía a través de un muro de bloque hueco de concreto, es tridimensional con los mecanismos de transferencia de calor acoplados; también tiene fronteras especiales en contacto con ambientes distintos y al menos una de ella con variación de condiciones en el tiempo. El presente trabajo considera las siguientes hipótesis para la solución numérica del problema:

1. La transferencia de calor a través del bloque hueco de concreto y del aislamiento térmico es en estado permanente.

2. Las fronteras fría y caliente son isotérmicas y todas las demás adiabáticas (fig. 3).

3. La transferencia de calor se resuelve de forma bidimensional en el caso de la conducción y la convección y unidimensional en el caso de la radiación (fig. 4).

4. La conductividad térmica del concreto es uniforme y constante (medio isotrópico).

La conducción bidimensional en el concreto se resuelve utilizando la siguiente ecuación:

$\frac{\partial T^{2}(x, y)}{\partial x^{2}}+\frac{\partial T^{2}(x, y)}{\partial y^{2}}$

El flujo de calor se calcula de acuerdo con la Ley de Fourier, donde:

$q_{x}=-\lambda \frac{\partial T(x, y)}{\partial x}$ y $q_{y}=-\lambda \frac{\partial T\left(x_{r} y\right)}{\partial y}$ 
Frontera isotérmica fría

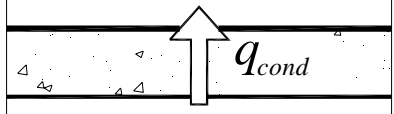

cavidad

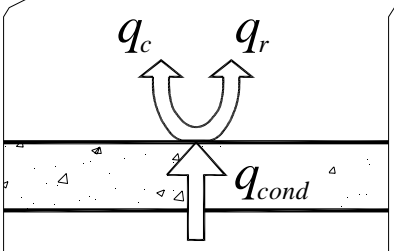

Frontera isotérmica caliente
Frontera isotérmica fría

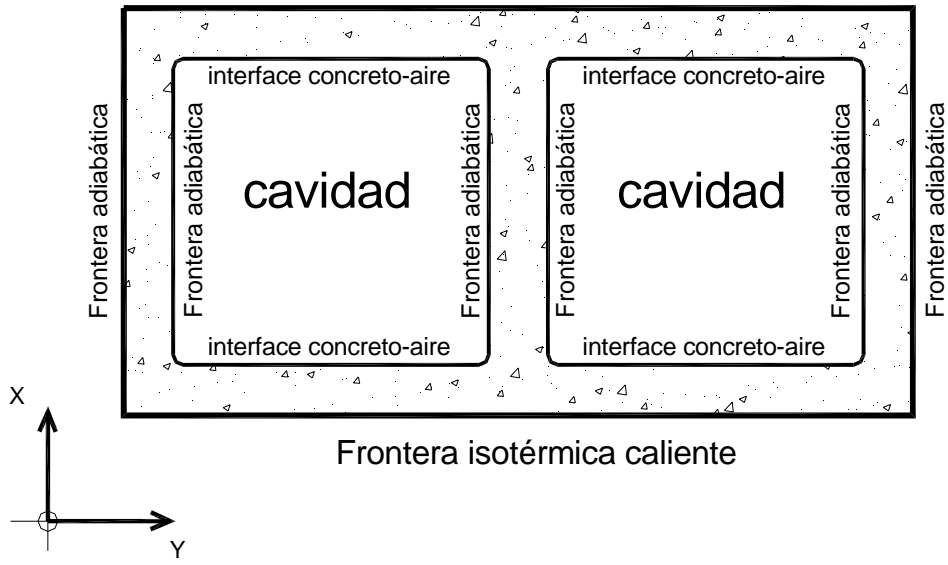

Fig. 3: Transporte de calor a través de la cavidad del bloque.
Fig. 4: Condiciones de frontera del bloque de concreto.

El transporte de energía por convección natural en la cavidad del bloque se determina con la ley de enfriamiento de Newton, considerando un espacio cerrado de forma rectangular vertical y el aire como fluido térmico (fig. 5).

$q_{e}=-h_{e} A\left(T_{h}-T_{c}\right)$

El coeficiente convectivo $\left(h_{c}\right)$ se obtiene con la expresión:

$\boldsymbol{h}_{v}=\frac{\lambda_{\text {airt }} N_{u s}}{L_{c}}$

Para el cálculo del número de Nusselt se utilizó la correlación de ElSherbiny (1982):

$N u_{1}=0.0605 R a_{L}^{\frac{1}{3},} \quad N u_{2}=\left[1+\left\{\frac{0.104 R a_{L}{ }^{0.798}}{1+\left(\frac{0310}{R a_{L}}\right)^{1.36}}\right\}^{3}\right], N u_{3}=0.242\left(\frac{R a_{L}}{\left(\frac{H}{L}\right)}\right)^{0.272}$

$N u=\max \left(N u_{1}, N u_{z}, N u_{3}\right)$

Aplicable para $R a_{L} \leq 2 \times 10^{6}$

En esta correlación las propiedades termofísicas del fluido se evalúan a la temperatura promedio de la cavidad.

El transporte de energía por radiación en las cavidades, se resuelve considerando el intercambio radiativo entre dos superficies planas de longitud infinita (fig. 6). En forma matemática:

$q_{r}=-h_{r} A\left(T_{h}-T_{v}\right)$

Donde:

$h_{r}-\varepsilon_{\mathrm{e}} \sigma\left(T_{h}+T_{c}\right)\left(T_{h}{ }^{2}+T_{c}{ }^{2}\right)$

$T_{c}$ y $T_{h}$ son las temperaturas de las paredes interiores del hueco en Kelvin, $h_{r}$ es el coeficiente radiativo, $A$ es el área perpendicular al flujo de calor, $\sigma$ es la constante de Stefan-Boltzmann y $\varepsilon_{e}$ es la emisividad efectiva entre las placas. 


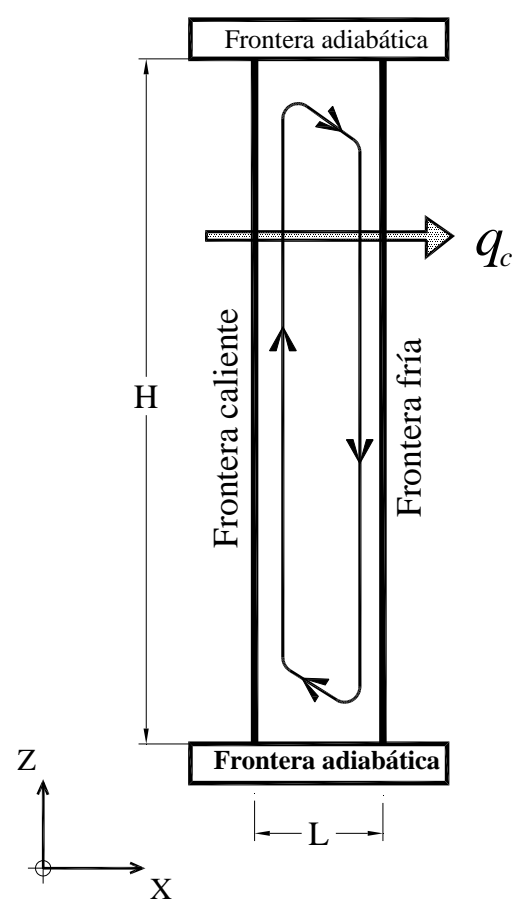

Fig 5, Esquema de convección natural en una cavidad cerrada

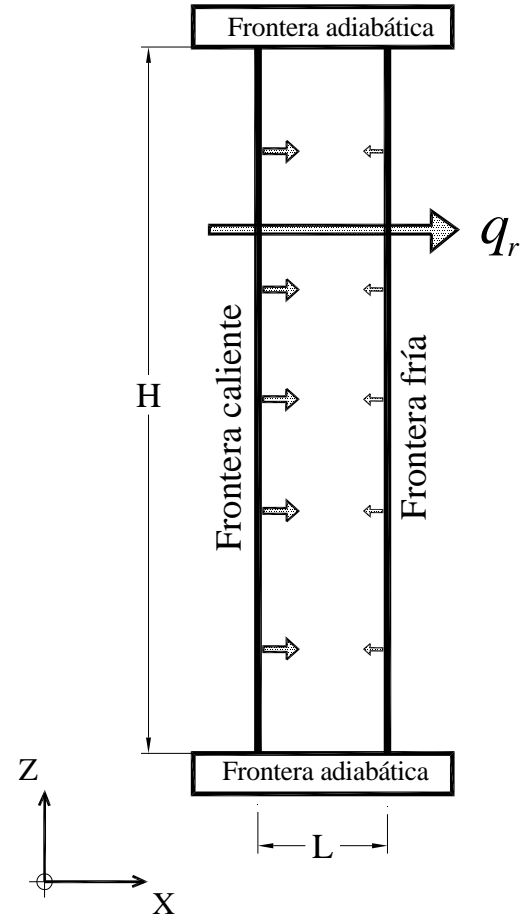

Fig 6, Esquema de intercambio radiativo en una cavidad cerrada

\section{SOLUCIÓN NUMÉRICA DEL MODELO DE TRANSFERENCIA DE CALOR.}

Las ecuaciones diferenciales para la conducción de calor, se discretizan para obtener ecuaciones algebraicas lineales, con el método de diferencias finitas; también se desarrollan ecuaciones especiales según las condiciones de frontera y de interface, como se describe a continuación:

Nodos internos (fig. 7):

$T_{i, j}=\frac{T_{i-1, j}+T_{i, j-1}+T_{i+1, j}+T_{i, j+1}}{4}$

Frontera adiabática (fig. 8):

$T_{i, j}=0.25 T_{i, j-1}+0.5 T_{i+1, j+0.25} T_{i, j+1}$

Interface de los materiales sólidos (fig. 9):

$T_{i, j}=\frac{\lambda_{1} T_{i-1, j+} \lambda_{2} T_{i+1, j+} \lambda_{p r o m}\left(T_{i, j-1+} T_{i, j+1}\right)}{2\left(\lambda_{2}+\lambda_{2}\right)}$

Dónde: $\lambda_{\text {prom }}=\frac{\lambda_{1}+\lambda_{2}}{2}, \lambda_{1}$ y $\lambda_{2}$ son las conductividades térmicas del concreto y del aislante térmico respectivamente.

Interface sólido-aire (fig. 10):

$T_{i, j}=\frac{\lambda T_{i, !-1}+R T_{n, j}\left(h_{o}+h_{r}\right)}{1+R\left(h_{c}+h_{r}\right)}$ 


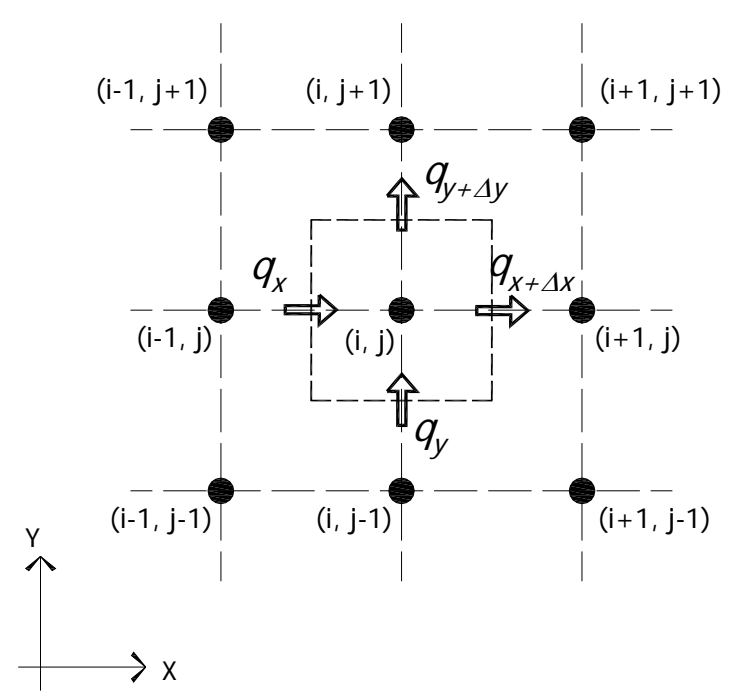

Fig. 7: Nodo interno de un material continuo y homogéneo.

Dónde:

$R=\frac{\boldsymbol{\Delta}}{\lambda} \mathbf{y} q_{x+\Delta x}=q_{e} \mid q_{r}$

Los cálculos se llevaron a cabo en hoja electrónica con un espaciamiento de malla uniforme y constante de $0.001 \mathrm{~m}$, resolviendo en forma iterativa el balance de energía en cada nodo. Los valores de temperatura de la superficie exterior del muro se encuentran en el intervalo $30^{\circ} \mathrm{C}-70^{\circ} \mathrm{C}$, de acuerdo a las observadas en un día típico de verano en la Ciudad de Hermosillo, Sonora, México. En la superficie fría del muro se consideran condiciones de temperatura constante a 25 ${ }^{\circ} \mathrm{C}$, equivalentes a las de un espacio climatizado artificialmente. Para las propiedades térmicas y ópticas del concreto, se usan los valores reportados por Cengel (2003) $\left(\lambda_{\text {concreto }}=1.10 \mathrm{~m}^{2} \mathrm{~K} / \mathrm{W}\right.$ y $\varepsilon_{\text {concreto }}=0.93$ ).

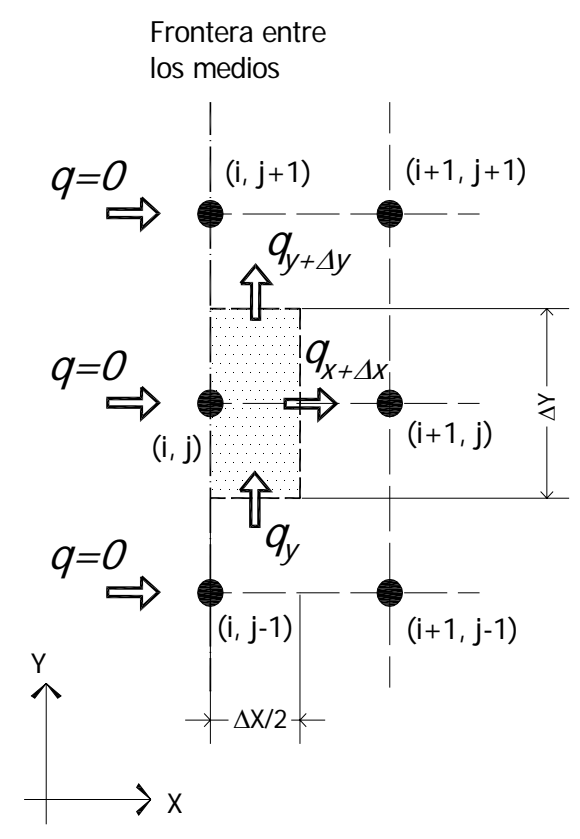

Fig. 8: Frontera adiabática $(q=0)$. 


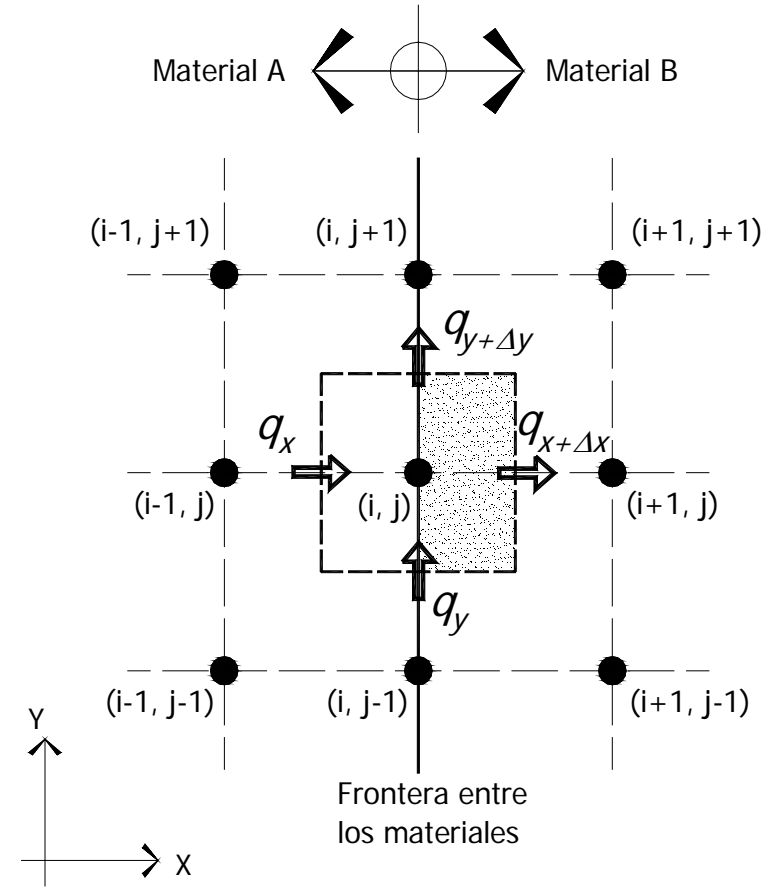

Fig. 9: Interface entre dos materiales.

\section{DISCUSIÓN DE RESULTADOS}

Para validar el modelo matemático y el algoritmo numérico, se realizó una comparación con los datos experimentales reportados en la literatura por Ossama (1994) y Borbón A. (2010), para lo cual se consideraron las condiciones experimentales empleadas en cada caso. La Tabla 1 resume las condiciones y los resultados de la comparación sin considerar el efecto de la unión entre bloques con mortero. Con base en las diferencias porcentuales obtenidas (entre $4.83 \%$ para el bloque de $15 \mathrm{~cm}$ y $6.32 \%$ para el bloque de $12 \mathrm{~cm}$ ), el modelo matemático y el algoritmo numérico se consideraron como validados.
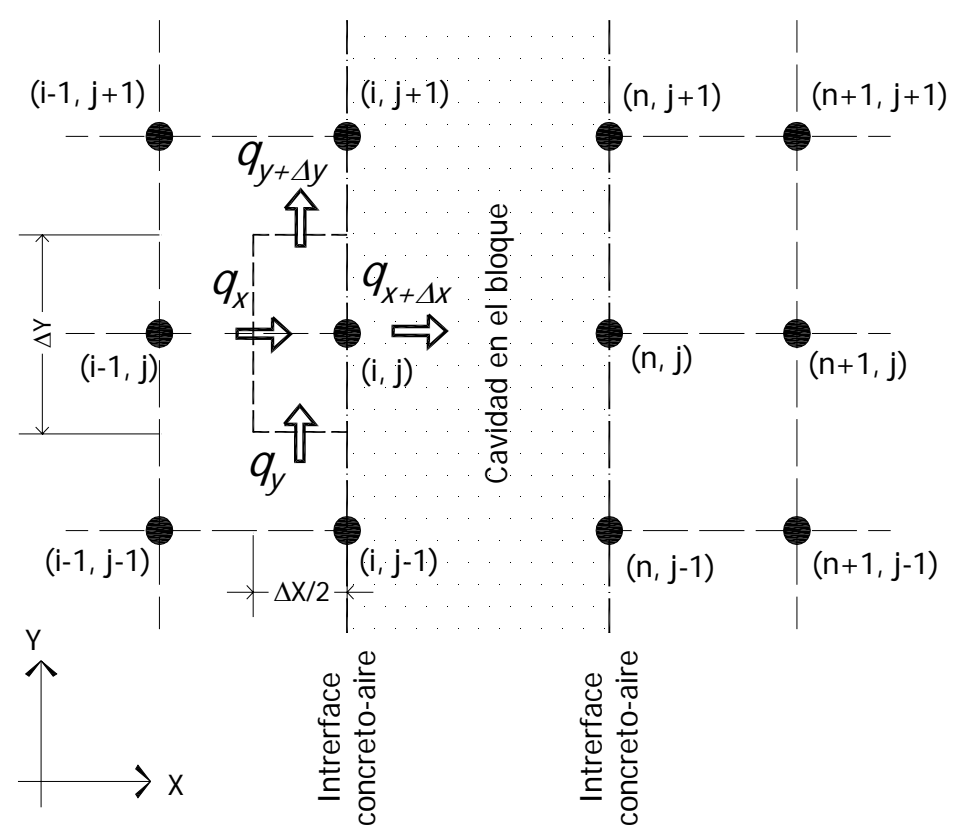

Fig. 10: Interface concreto-aire. 
Tabla 1: Comparación de los resultados teóricos con experimentales.

\begin{tabular}{|l|c|c|c|c|c|c|c|}
\hline & & & & \multicolumn{3}{|c|}{ Resistencia térmica del muro } & \\
\cline { 5 - 8 } $\begin{array}{l}\text { Descripción del } \\
\text { muro }\end{array}$ & $T_{S C}$ & $T_{S F}$ & $\Delta T$ & \multicolumn{2}{|c|}{$\left(\mathrm{m}^{2} \mathrm{~K} / \mathrm{W}\right)$} & $\begin{array}{c}\text { Error relativo } \\
(\%)\end{array}$ \\
\cline { 5 - 8 } & $(K)$ & $(K)$ & $(K)$ & Ossama & Borbón & $\begin{array}{l}\text { Trabajo } \\
\text { actual }\end{array}$ & 4.83 \\
\hline $\begin{array}{l}\text { Muro con bloque } 15 \mathrm{~cm} \\
\text { de }\end{array}$ & 54.9 & 29.0 & 25.0 & 0.331 & ----- & 0.347 & 6.32 \\
\hline $\begin{array}{l}\text { Muro con bloque } \\
\text { de 12 cm }\end{array}$ & 28.76 & 42.34 & 13.58 & ------ & 0.174 & 0.163 & 6 \\
\hline
\end{tabular}

Una vez realizada la validación, se obtuvieron resultados para el muro considerando bloques de concreto hueco de 12 y $15 \mathrm{~cm}$, para diferencias de temperaturas de $5 \mathrm{~K}$ hasta $45 \mathrm{~K}$. En la Figura 11 se presenta la variación de la resistencia térmica en el rango de diferencias de temperatura considerado, para las dos configuraciones de bloque hueco estudiadas. Se aprecia que los valores de la resistencia térmica para el muro con bloques de $12 \mathrm{~cm}$ se encuentran entre 0.190 $\mathrm{m}^{2} \cdot \mathrm{K} / \mathrm{W}(\Delta T=5 \mathrm{~K})$ y $0.158 \mathrm{~m}^{2} \cdot \mathrm{K} / \mathrm{W}(\Delta T=45 \mathrm{~K})$, con un valor promedio de $0.159 \mathrm{~m}^{2} \cdot \mathrm{K} / \mathrm{W}(\Delta T=5 \mathrm{~K})$; mientras que en el caso del bloque de $15 \mathrm{~cm}$, los valores de la resistencia térmica son ligeramente menores (entre $8.4 \%$ y $5.7 \%$ respectivamente), ya que van de $0.174 \mathrm{~m}^{2} \cdot \mathrm{K} / \mathrm{W}(\Delta T=5 \mathrm{~K})$ a 0.149 $\mathrm{m}^{2} \cdot \mathrm{K} / \mathrm{W}(\Delta T=45 \mathrm{~K})$, con un valor promedio de la resistencia térmica de $0.171 \mathrm{~m}^{2} \cdot \mathrm{K} / \mathrm{W}$.

La disminución de la resistencia térmica del muro de bloque de concreto con el aumento del gradiente térmico, se debe a la variación de las propiedades termofísicas del fluido (aire) con la temperatura en el interior de la cavidad alargada que forman los bloques. Esta disminución resulta inconveniente, ya que el valor más bajo de la resistencia térmica se presenta en las condiciones más adversas para el confort térmico en el interior de las edificaciones.

Con los resultados se obtuvieron las siguientes correlaciones utilizando la técnica de regresión por mínimos cuadrados, para la variación de la resistencia térmica del muro de bloque de concreto con la diferencia de temperatura:

$$
\begin{array}{ll}
R_{12 \mathrm{~cm}}=0.1858-0.0055 \Delta T^{\frac{1}{2}} & \left(r^{2}=0.998\right) \\
R_{15 \mathrm{~cm}}=0.2041-0.0069 \Delta T^{\frac{1}{2}} & \left(r^{2}=0.993\right)
\end{array}
$$

Debido a que la resistencia térmica del muro de bloque resulta muy baja, se estudió el comportamiento de éste sistema constructivo con aislamiento térmico (poliestireno) en la superficie exterior (sin aplicar ningún tipo de recubrimiento) o rellenando las cavidades. Las figuras 12 y 13 muestran el comportamiento de la resistencia térmica para los bloques de $12 \mathrm{~cm}$ y $15 \mathrm{~cm}$ respectivamente. Para facilitar la comparación se utiliza el mismo volumen de aislamiento térmico en las dos opciones consideradas. Se aprecia una diferencia significativa en los valores de la resistencia térmica como efecto de la posición del aislante térmico. En la figura 12, se observa que cuando se aplica un espesor de aislamiento térmico de $25 \mathrm{~mm}$ en la superficie exterior, la resistencia térmica del muro es igual $0.776 \mathrm{~m}^{2} \cdot \mathrm{K} / \mathrm{W}$, sin embargo, cuando se coloca el mismo volumen de aislante térmico en la cavidad, la resistencia térmica es de $0.168 \mathrm{~m}^{2} \cdot \mathrm{K} / \mathrm{W}$, lo que representa una diferencia porcentual de $361 \%$.

En general para ambos bloques, cuando el aislante térmico se coloca en la superficie exterior la resistencia térmica del muro aumenta de forma significativa, mientras que al rellenar las cavidades la resistencia térmica no se tiene un incremento apreciable (excepto cuando se utiliza un volumen de aislamiento equivalente a un espesor de $58 \mathrm{~mm}$ en la superficie exterior). Lo anterior se debe a que al colocar el aislamiento en la superficie exterior, se evita que la pared exterior del bloque se sobrecaliente, lo que disminuye sustancialmente la transferencia de calor por conducción en el 
concreto, así como por convección y radiación en la cavidad; mientras que al rellenar la cavidad se suprimen la convección y la radiación, pero no la conducción a través del concreto.

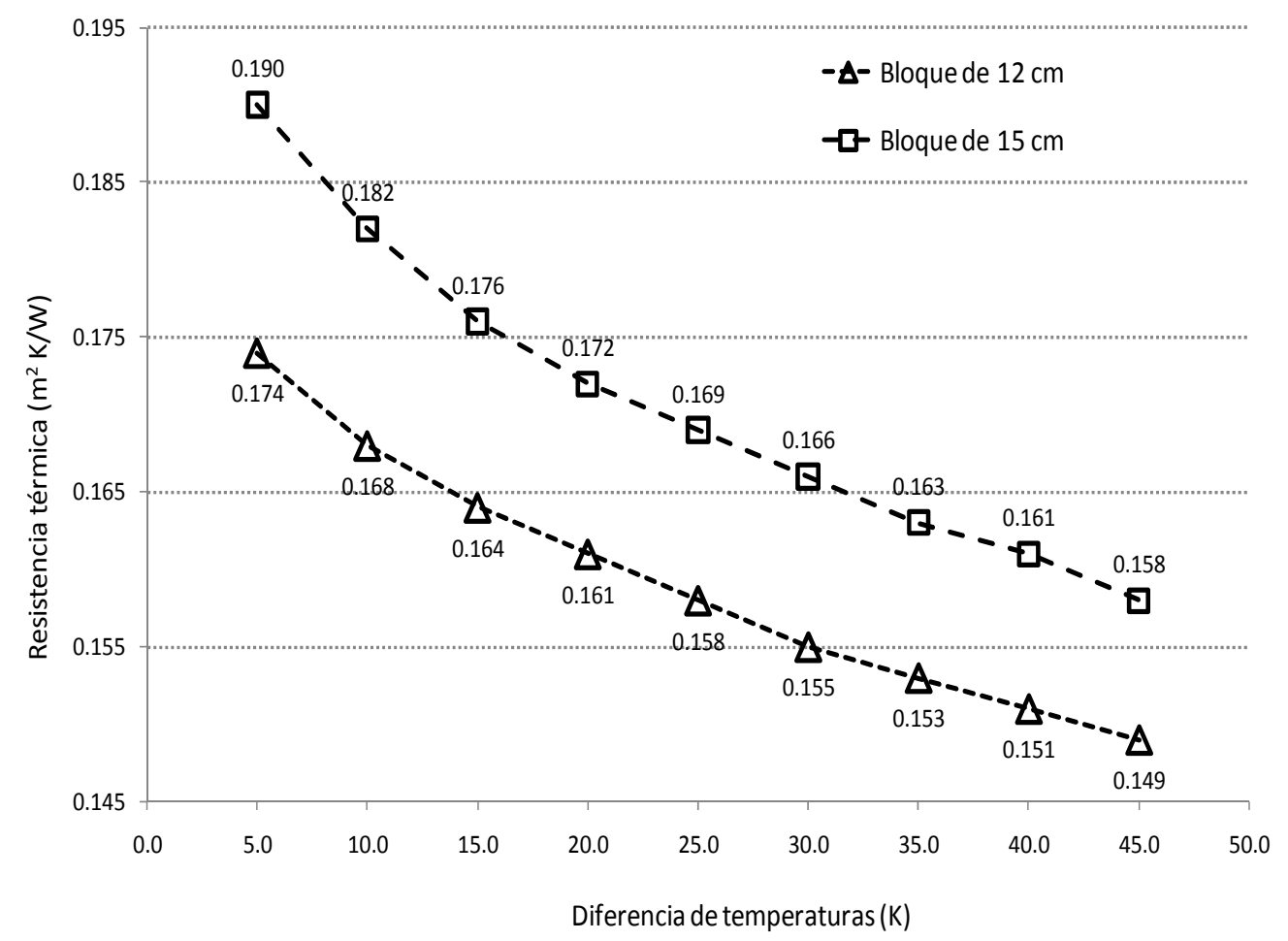

Fig. 11, Variación de la resistencia térmica de los bloques de concreto con el gradiente térmico.

Las correlaciones para la variación de la resistencia térmica del muro de bloque de concreto con el espesor de aislamiento $\left(e_{a}\right)$ son las siguientes:

Muro de bloque de concreto con aislamiento térmico en la superficie exterior

$R_{1 \mathrm{~cm}}=0.1604-0.0244 e_{\mathrm{a}}\left(r^{2}=0.999\right)$

$R_{15 \mathrm{~cm}}=0.1716-0.0245 \varepsilon_{\mathrm{a}}\left(r^{2}=0.999\right)$

Muro de bloque de concreto con aislamiento térmico en la cavidad

$$
\begin{array}{ll}
R_{1 \mathrm{am}}=\frac{0.1595-.0026 e_{a}}{1-0.0170 e_{a}} & \left(r^{2}=0.998\right) \\
R_{1 \overline{a m}}=\frac{0.1596-.0019 e_{a}}{1-0.0162 e_{a}} & \left(r^{2}=0.990\right)
\end{array}
$$

\section{CONCLUSIONES}

Con base en los resultados obtenidos, se puede concluir lo siguiente:

1. La resistencia térmica del bloque hueco de concreto disminuye cuando aumenta el gradiente térmico, lo anterior es un inconveniente ya que se tiene una menor oposición a la transferencia de calor, cuando se tiene un mayor sobrecalentamiento en el ambiente.

2. La resistencia térmica de un muro de bloques huecos de concreto, se incrementa linealmente en función del espesor del aislamiento térmico, cuando se aplica en la cara exterior.

3. La resistencia térmica del bloque cuando se aplica aislamiento térmico dentro de la cavidad tiene un valor límite de $0.407 \mathrm{~m}^{2} \mathrm{~K} / \mathrm{W}$ y de $0.530 \mathrm{~m}^{2} \mathrm{~K} / \mathrm{W}$ para el bloque de 12 y $15 \mathrm{~cm}$ respectivamente; mientras que para el mismo volumen de aislante térmico en la cara exterior del 
bloque, la resistencia térmica es de $1.555 \mathrm{~m}^{2} \mathrm{~K} / \mathrm{W}$ y $2.147 \mathrm{~m}^{2} \mathrm{~K} / \mathrm{W}$, para el bloque de 12 y $15 \mathrm{~cm}$ respectivamente.

4. Es más conveniente aplicar el aislamiento térmico por la cara exterior del bloque, ya que los valores máximos de resistencia térmica rellenando las cavidades, son alrededor del $25 \%$ de los valores máximos de resistencia térmica aplicando el mismo volumen de aislamiento en la cara exterior del bloque de concreto hueco.

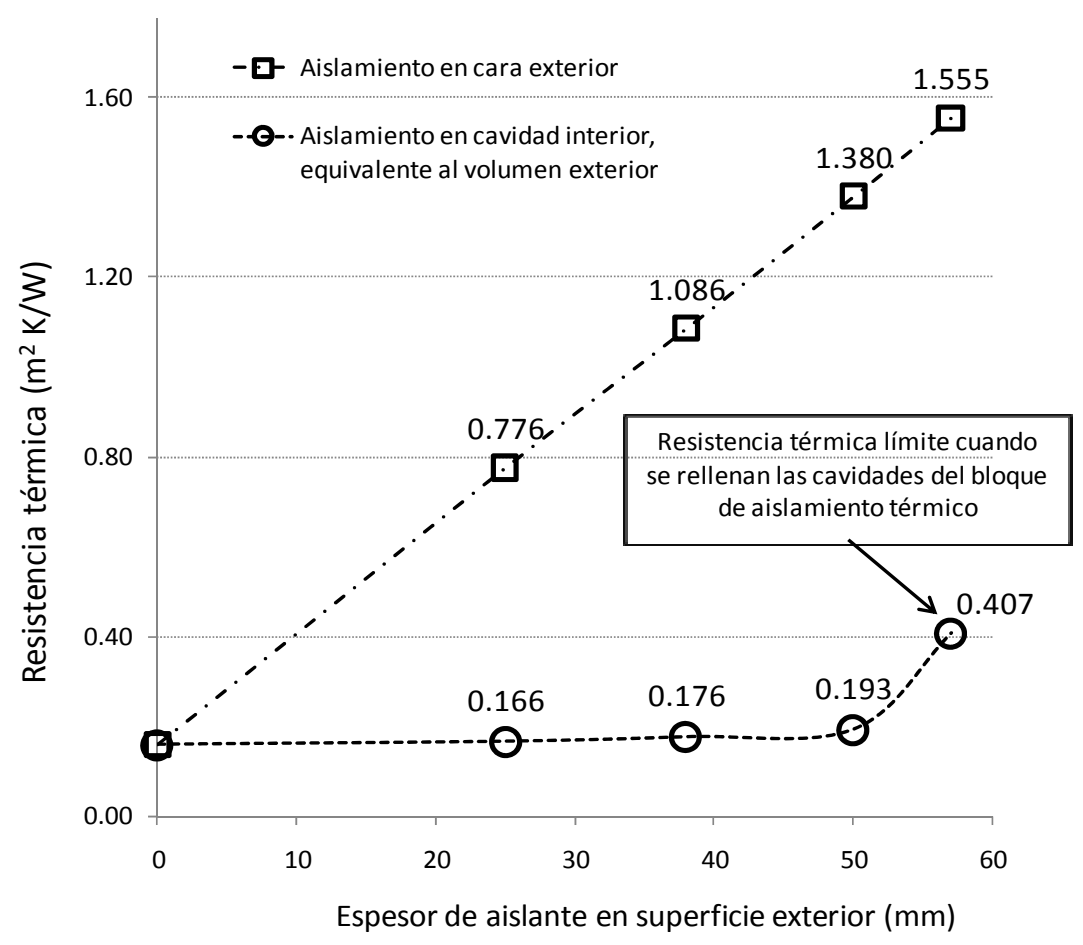

Fig. 12: Resistencia térmica con aislante térmico exterior y en huecos; bloque de $12 \mathrm{~cm}$.

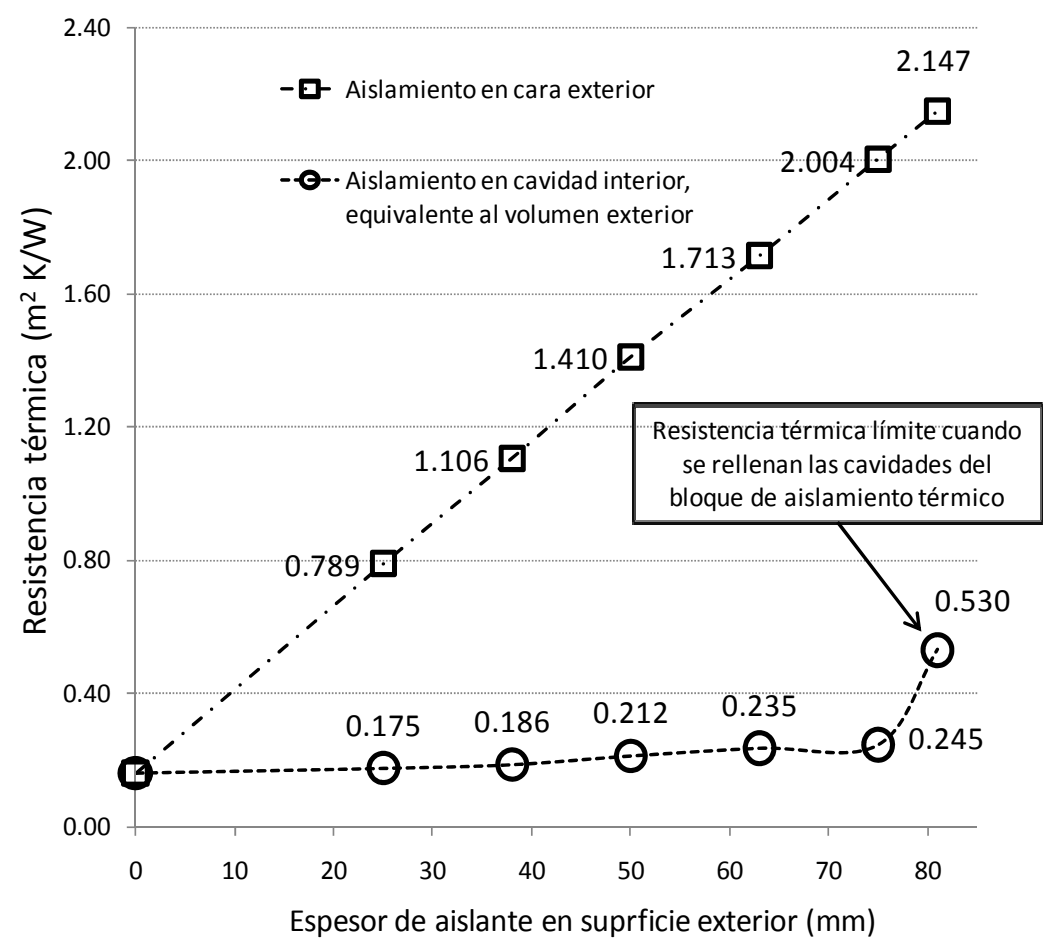

Fig. 13: Resistencia térmica con aislante térmico exterior y en huecos; bloque de $15 \mathrm{~cm}$. 


\section{NOMENCLATURA}

$q_{x}, q_{y} \quad$ Flujo de calor en las direcciones $x$ e y por unidad de área $\left(\mathrm{W} / \mathrm{m}^{2}\right)$

$q_{c}, q_{r} \quad$ Flujo de calor convectivo y radiativo respectivamente por unidad de área $\left(\mathrm{W} / \mathrm{m}^{2}\right)$

A Área transversal de transferencia de calor $\left(\mathrm{m}^{2}\right)$

$h_{c,} h_{r} \quad$ Coeficiente convectivo y radiativo respectivamente $\left(\mathrm{W} / \mathrm{m}^{2} \cdot \mathrm{K}\right)$

$T_{h} \quad$ Temperatura de la superficie caliente de la cavidad (K)

$T_{c} \quad$ Temperatura de la superficie fría de la cavidad (K)

$\mathrm{Nu} \quad$ Número de Nusselt

$L_{c} \quad$ Longitud característica de la cavidad (m)

$\lambda_{\text {aire }} \quad$ Conductividad térmica del aire $(\mathrm{W} / \mathrm{m} \cdot \mathrm{K})$

$\lambda_{x}, \lambda_{y} \quad$ Conductividad térmica del material en cada dirección espacial (W/m/K)

$P_{r} \quad$ Número de Prandtl

$R a_{L} \quad$ Número de rayleigh

$T(x, y) \quad$ Temperatura del material (K)

$T_{i, j} \quad$ Temperatura del nodo $\mathrm{i}, \mathrm{j}(\mathrm{K})$

$\Delta T \quad$ Diferencia de temperaturas $(\mathrm{K})$

$\sigma \quad$ Constante de Stefan-Boltzmann $\left(\mathrm{W} / \mathrm{m}^{2} \cdot \mathrm{K}^{4}\right)$

$\varepsilon_{\mathrm{e}} \quad$ Emisividad efectiva entre las placas $=\frac{1}{\frac{1}{\varepsilon_{1}}+\frac{1}{\varepsilon_{2}}-1}$

\section{AGRADECIMIENTOS}

Los autores agradecen a la Universidad de Sonora, el apoyo para la publicación de este artículo.

\section{REFERENCIAS}

Anderson, B. R., On the Calculation of the U-value of Walls Containing Slotted Bricks or Blocks, Building and Environment: 16 (1), 41-50 (1981).

Aviram, D.P., A.N. Fried, J.J. Roberts, Thermal Properties of a Variable Cavity Wall, Building and Environment: 36, 1057-1072 (2001).

Borbón A. Ana, Cabanillas L. Rafael, Pérez V. Jesús, "Determinación experimental y contraste numérico de la resistencia térmica de un muro de bloques de concreto hueco", Información Tecnológica, 6 Vol. 21 (2010). 
Elsherbiny, S.M. G.D. Raithby, K.G.T. Hollands, Heat Transfer by Natural Convection across Vertical and Inclined Air Layers, Journal of Heat Transfer: Transactions of the ASME 104. 96-102 (1982).

Lacarrie're B. Numerical Study of Heat Transfer in a Wall of Vertically Perforated Bricks: Influence of Assembly Method, Energy and Buildings: 35, 229-237 (2003).

Lacarrie're B.Trombe A., Monchoux F.,Experimental Unsteady Characterization of Heat Transfer in a Multi-Layer Wall Including Air Layers-Application to Vertically Perforated Bricks, Energy and Buildings: 38, 232-237 (2006).

Lorente S., M. Petit, R. Javelas, Simplified Analytical Model for Thermal Transfer in Vertical Hollow Brick,Energy and Buildings: 24, 95-103(1996).

Ossama A. Abdou, Kris S. Murali b., The Efect of Air Cells and Mortar Joints on the Thermal Resistance of Concrete masonry Walls, Energy and Buildings: 21,111-119 (1994).

Majed M. Al-Hazmy. Analysis of coupled natural convection-conduction effects on the heat transport through hollow building blocks, Energy and Buildings: 38, 515-521 (2006).

Vasile C., Lorente S., B. Perrin, Study of convective phenomena inside cavities coupled with heat and

mass transfers through porous media, Energy and Buildings: 28, 229-235 (1998)

Wakili K. Ghazi, Tanner Ch., U-value of Dried Wall Made of Perforated Porous Clay Bricks Hot Box Measurement Versus Numerical Analysis, Energy and Buildings: 35, 675-680 (2003). 